\title{
Internationalisierung von Dienstleistungen - Die EU-Dienstleistungsrichtlinie
}

Zur EU-Dienstleistungsrichtlinie werden in der Literatur völlig konträre Standpunkte vertreten. Heißt das, einige Autoren müssen schlimmen Irrtümern aufgesessen sein? Nein - dieser Aufsatz zeigt, dass sich in der Debatte schlicht unterschiedliche Perspektiven widerspiegeln. Beispielsweise werden die Vorteile intensiverer internationaler Arbeitsteilung insgesamt zu höherer Dienstleistungsbeschäftigung führen und außerdem zu Vorteilen für die Konsumenten. Dieser positiven gesamtwirtschaftlichen Einschätzung zum Trotz kann man widerspruchsfrei befürchten, dass infolge der Richtlinie für bestimmte Gruppen von Arbeitnehmern vermehrte arbeitsmarktpolitische Unterstützung nötig wird.

\section{Einleitung}

Wenn nicht im Dienstleistungssektor Arbeitsplätze geschaffen werden, wo dann? Die wichtigsten Impulse, die das verarbeitende Gewerbe noch zu geben vermag, bestehen sicherlich darin, dass es Dienstleistungen nachfragt und somit indirekt - statt direkt in der Produktion - Arbeitsplätze schafft. Hiervon abgesehen steht der Dienstleistungssektor unter großem Einfluss des Staates, der selbst Dienstleistungen anbietet oder - wie im Gesundheitssektor - ihre Finanzierung gestaltet und damit unmittelbar die Grenzen ihres Wachstums bestimmt. Die EU übt nun ebenfalls einen bedeutenden (wenngleich weniger diskretionären, sondern mehr ordnungspolitischen) Einfluss aus. Die EU-Dienstleistungsrichtlinie muss nach ihrer Verabschiedung durch das Europäische Parlament im November 2006 bis 2010 in nationales Recht umgesetzt werden. Damit wäre dann - mit Ausnahme einiger Branchen ${ }^{1}$ der freie Dienstleistungsverkehr zwischen den Mitgliedstaaten als eine der vier Grundfreiheiten des Binnenmarktes erreicht.

Der dazu beschrittene Weg war bis zuletzt umstritten wie kaum ein anderes EUProjekt. Anfang 2004 war der Richtlinienentwurf von der EU-Kommission vorgelegt worden; den europäischen Gewerkschaften gelang am 19. März 2005 die Mobilisierung von 60.000 Demonstranten in Brüssel, was viel ist, angesichts der Tatsache, dass es nicht unmittelbar um Tarifverträge oder Entlassungen ging. Die Regierungen von Deutschland und Frank- reich zeigten sich beeindruckt und distanzierten sich vom Richtlinienentwurf, den sie als „,sozialpolitisch unausgewogen“ (Lorenz/Wannöffel 2006, S. 17) bezeichneten. Auch für den in der Folge mühsam ausgehandelten Kompromiss ist die Spanne der Bewertungen groß: von „katastrophal“ bis „heilbringend“ lauten die Zuschreibungen. Wie kann das sein?

Erstens können diese Unterschiede auf einfache Fehlinformationen oder gedankliche Fehler zurückgehen, was aber in der jetzigen Phase der Diskussion niemandem mehr unterstellt werden sollte. Zweitens kann es unterschiedliche Erwartungen über künftige Entwicklungen geben, die einen Einfluss auf die Wirkung der Dienstleistungsrichtlinie haben und die objektiv unsicher sind. Hierfür werden im Folgenden einige Beispiele benannt. Und drittens kann es sein, dass schlicht die Perspektive entscheidet, genauer gesagt: das Kriterium, das dem Urteil zugrunde liegt. Je nachdem, ob die Perspektive juristisch, international, makroökonomisch oder verteilungspolitisch ist, erscheint die Richtlinie ohne gedanklichen Fehler als

- in ihrer Bedeutung überschätzt,

- überfällig,

- Chance für den Arbeitsmarkt und für die Konsumenten oder

- Bedrohung für kritische Teile des Arbeitsmarktes.

\section{Die juristische Perspektive}

Die Dienstleistungsrichtlinie soll und wird dazu führen, dass viele Anbieter von
Dienstleistungen sich plötzlich neuer Konkurrenz aus vielen EU-Staaten gegenübersehen. Insofern könnte man die Richtlinie tatsächlich als revolutionär bezeichnen. Um die Schärfe aus der Diskussion zu nehmen, ist es aber nützlich, darauf hinzuweisen, dass die Richtlinie nichts anderes ist als ein Instrument zur Umsetzung längst geltenden EU-Rechts (Eichenhofer 2006). Als Beispiel ist hier die Niederlassungsfreiheit zu nennen. Die Dienstleistungsrichtlinie schreibt erhebliche Verwaltungsvereinfachungen vor und ermöglicht so eine diskriminierungsfreie Niederlassung ausländischer Anbieter - im Sinne einer der vier Grundfreiheiten des Binnenmarktes.

Im Zentrum der öffentlichen Diskussion steht allerdings der grenzüberschreitende Dienstleistungshandel, der auf eine andere Grundfreiheit, die Dienstleistungsfreiheit, zielt (Art. 49 EG-Vertrag). Dass es noch bestehende Einschränkungen freien internationalen Handels mit Dienstleistungen gibt, die alle gegen den EG-Vertrag verstoßen, zeigen folgende Beispiele (aus Nerb et al. 2006, nach Angaben der IHK München):

\footnotetext{
1 Beispielsweise Finanzdienstleistungen, soziale Dienstleistungen, audiovisuelle Dienste, Abfallentsorgung, Glücksspiel, Verkehrsdienstleistungen, Dienstleistungen von Notaren und Leiharbeitsagenturen.
} 
Beispiel 1: Anerkennungsverfahren nach der österreichischen Ingenieurkonsulentenverordnung

Ein bayerisches Ingenieurbüro führt regelmäßig Messarbeiten für Tunnelbauten in Österreich durch. Für jeden Auftrag muss es vorab ein Anerkennungsverfahren nach der österreichischen EWR-Ingenieurkonsulentenverordnung durchlaufen. Die erteilte Anerkennung gilt nicht dauerhaft, sondern immer nur für einen Auftrag. Da die Bearbeitungszeit bei den österreichischen Behörden mehrere Wochen betragen kann, besteht immer die Gefahr, dass Aufträge wieder entzogen werden.

\section{Beispiel 2: Genehmigung für Montage- arbeiten in Luxemburg}

Ein deutscher Bauhandwerker benötigt für Montagearbeiten in Luxemburg eine Genehmigung, auf deren Ausstellung er mehrere Monate warten muss. Um überhaupt rechtzeitig tätig werden zu können, muss er einen attraktiven Teil des Auftrags an einen luxemburgischen Subunternehmer abgeben.

Beispiel 3: Medizinische Untersuchung und Sicherheitskurse in Großbritannien

Eine deutsche Firma führt regelmäßig Arbeiten in Großbritannien im Bereich Bahngleisuntersuchungen durch. Um die Arbeiten ausführen zu können, muss sich das Schlüsselpersonal der Firma einem medizinischen Test unterziehen und an einem mehrtägigen Sicherheitstraining teilnehmen. Die medizinische Untersuchung muss zwingend in Großbritannien erfolgen. Untersuchungsberichte deutscher Ärzte werden nicht anerkannt. Die medizinische Untersuchung und das Sicherheitstraining können außerdem nicht im Zuge eines Aufenthalts in England erfolgen, weil der Sicherheitstest erst absolviert werden darf, wenn der Medizintest positiv verlaufen ist. Das Sicherheitstraining kann ebenfalls nicht im Zusammenhang mit der Ausführung des Auftrags durchgeführt werden, weil der Auftrag erst nach einem erfolgreich absolvierten Sicherheitstraining erteilt werden kann. Für die medizinische Untersuchung und das Sicherheitstraining muss das Schlüsselpersonal der deutschen Firma folglich mehrfach nach England reisen, bevor der eigentliche Auftrag beginnt.
Beispiel 4: Prüfungen zum staatlich anerkannten Tourismus-Führer in Österreich und Italien

Deutsche dürfen in Italien und Österreich nur Reisegruppen führen, wenn sie eine aufwendige Prüfung als staatlich anerkannte Reiseführer ablegen; dies gilt selbst bei Tagesausflügen, die in Deutschland beginnen und enden.

Beispiel 5: CFK-Prüfung für den Bereich Kühlungstechnik in den Niederlanden

Eine bayerische Firma hatte einen Auftrag bei der niederländischen Armee für den Einbau eines Kühlsystems gewonnen. Der Geschäftsinhaber, ein erfahrener Meister im Kühlanlagenbau, darf jedoch nur grenzüberschreitend als Kälteanlagenbauer arbeiten, wenn er eine mehrwöchige Schulung mit anschließender Prüfung in niederländischer Sprache absolviert.

Wenn man bedenkt, dass all diese Hemmnisse durch Klage beim Europäischen Gerichtshof aus der Welt zu schaffen wären, und dass die Dienstleistungsrichtlinie nur ein alternativer, einfacherer Weg ist, dies zu tun, dann könnte es so aussehen, als ob die Richtlinie in ihrer Bedeutung etwas überschätzt wird. Allerdings ist der Klageweg mühsam und auf Einzelfälle beschränkt. Die Dienstleistungsrichtlinie stellt hingegen in einem Handstreich einen Zustand her, für den es sonst Jahrzehnte des Prozessierens bedurft hätte. Der Handstreich hatte in der ersten Fassung der Richtlinie einen Namen: Herkunftslandprinzip. Wer dort, wo sein Unternehmen ansässig ist (Herkunftsland), eine bestimmte Dienstleistung anbieten darf, der soll dies auch in anderen EU-Ländern dürfen. Das heißt nicht, dass er in jeder Hinsicht die Gesetze des Bestimmungslandes ignorieren darf - beispielsweise gelten für ihn auch das Umweltrecht, das Verbraucherrecht und Teile des Arbeitnehmerschutzes des Bestimmungslandes. Aber erstmals darf der Exporteur von Dienstleistungen sich diskriminierungsfrei behandelt fühlen. „Herkunftslandprinzip“ ist allerdings so sehr zu einem Reizwort geworden, dass es aus der aktuell vorliegenden Formulierung der Richtlinie verbannt wurde. Jedoch scheint es sich letztlich nur um die Änderung der Formulierung zu handeln, während die Idee erhalten blieb. Ob dieser Eindruck täuscht und Einfallstore für neue Handels- hemmnisse offen geblieben sind, kann derzeit und an dieser Stelle nicht beurteilt werden.

\section{3 \\ Die internationale Perspektive}

Die Europäische Kommission legte 2002 einen Bericht über den „Stand des Binnenmarkts für Dienstleistungen "vor. Er ist eine Fundgrube für Fälle von Hemmnissen für den Dienstleistungshandel, ähnlich den aufgelisteten Beispielen. Zugleich macht der Bericht deutlich, dass die Kommission große Erwartungen an die Beseitigung solcher Hemmnisse knüpft. Dies wird nicht zuletzt dadurch betont, dass ausdrücklich ein Zusammenhang mit der LissabonAgenda hergestellt wird, also zum Ziel, die Europäische Union bis 2010 zum „wettbewerbfähigsten und dynamischsten wissensbasierten Wirtschaftsraum der Welt" (Europäische Kommission 2002, S. 5) zu machen. Es hat zwei Gründe, dass der mit der Lissabon-Strategie verbundene Ehrgeiz besonders auf die Dienstleistungen übertragen wird: Erstens wäre ein echter Binnenmarkt für Dienstleistungen noch etwas Neues, während das Potenzial für innereuropäische Außenhandelsliberalisierung bei Gütern praktisch schon ausgeschöpft ist. Zweitens wies die Europäische Kommission (2002, S. 61) darauf hin, dass 1999 der Anteil der Dienstleistungen am Außenhandel der EU nur bei etwa $22 \%$ lag, während ihr Anteil am Bruttoinlandsprodukt (BIP) und an der Beschäftigung in den meisten EU-Staaten an die $70 \%$ betrug. Das bedeutet: Obgleich die Dienstleistungen bereits die nationalen Märkte dominieren, bestimmen nach wie vor die Güter den Außenhandel. Aus dieser Sicht war die Dienstleistungsrichtlinie überfällig.

Es wäre allerdings verfehlt, das Wachstumspotenzial der Im- und Exporte von Dienstleistungen am „Benchmark“ des Außenhandels mit Gütern zu messen. Kox, Lejour und Montizaan (2004) von CРB (Centraal Planbureau, Netherlands Bureau for Economic Policy Analysis) versuchen eine realistischere Prognose. Sie schätzen dazu ein Gravitationsmodell, das heißt eine Regressionsgleichung, in der die unabhängige Variable der Dienstleistungshandel zwischen Paaren von Staaten ist; erklärende Variable ist unter anderem die 
Entfernung zwischen den Staaten. Zu den weiteren erklärenden Variablen gehören Sprachbarrieren und einige Regulierungen des Dienstleistungshandels.

Diese Schätzungen lassen erkennen, welchen Einfluss Regulierungen auf den Handel haben, und somit kann man auch vorhersagen, wie sich der internationale Handel mit Dienstleistungen entwickeln wird, wenn durch die Dienstleistungsrichtlinie einige der Handelshemmnisse beseitigt werden. Die Autoren prognostizieren auf diese Weise eine Zunahme des innereuropäischen Dienstleistungshandels zwischen 30 und $60 \%$. Nerb et al. (2006) haben argumentiert, dass dies den positiven Effekt der Dienstleistungsrichtlinie aus methodischen Gründen eher unterschätzt, ${ }^{2}$ andererseits beruhen die Schätzungen von Kox et al. (2004) auf dem alten Richtlinienentwurf mit dem Herkunftslandprinzip, also möglicherweise auf einer so kräftigen Verringerung der Handelshemmnisse, wie sie tatsächlich nicht erreicht wird. ${ }^{3}$ Nehmen wir an, dass diese Einflüsse auf die tatsächliche Entwicklung des Außenhandels einander mehr oder weniger ausgleichen, und nehmen wir ferner an, dass der innereuropäische Außenhandel mit Dienstleistungen um 50 \% steigt; der gesamte Außenhandel mit Dienstleistungen stiege dann um etwa $28 \%$. Dennoch würde - bei absolut gleichbleibendem Güterhandel - der Anteil der Dienstleistungen am EU-Außenhandel lediglich von $22 \%$ auf $26,5 \%$ steigen.

Die Feststellung, dass der Außenhandel mit Dienstleistungen sich zwar kräftig steigern ließe, die Bedeutung des internationalen Güterhandels aber nicht erreichen wird, ist nicht das Ergebnis bloßer „Ökonometrickserei“. Vielmehr liegt es in der Natur vieler Dienstleistungen, dass sie nicht so leicht wie Güter handelbar sind. In diesem Zusammenhang ist die Klassifikation der World Trade Organisation (WTO) (2001) hilfreich, die vier Modi des Außenhandels mit Dienstleistungen unterscheidet:

(1) cross-border supply: Dienstleistungen sind typischerweise nicht "lagerbar" und erfordern das Zusammenwirken von Anbieter und Nachfrager, und zwar zur selben Zeit („Uno-actu-Prinzip“) und am selben Ort. Es gibt einige Ausnahmen, für die eine Leistungserbringung über größere räumliche Entfernungen möglich ist. Beispiele hierfür sind E-Learning, Buchhaltung und
Datenverarbeitung. Die WTO schätzt den Anteil dieses Modus am Welthandel mit Dienstleistungen auf etwa $35 \%,{ }^{4}$ bezogen auf den innereuropäischen Dienstleistungshandel ist dieser Anteil sicher geringer. Dieser Modus weist die wenigsten Diskriminierungen auf; die Richtlinie wird hier nicht für erhebliches Wachstum sorgen können.

(2) Dies gilt auch für den zweiten, consumption abroad genannten Modus; hier reist der Nachfrager zum Anbieter. Die WTO setzt den Anteil dieses Modus am gesamten internationalen Dienstleistungshandel mit 10 bis $15 \%$ an; bedeutend ist insbesondere der Tourismus. Zu einer speziellen Form der Diskriminierung ausländischer Anbieter kann es bei der Übernahme von Kosten für Gesundheitsdienstleistungen im Ausland kommen. Die Richtlinie wird hieran nichts ändern, zumal der Gesundheitssektor in der neuesten Fassung der Richtlinie - anders als im ersten Entwurf von 2004 - ohnehin nicht mehr erfasst ist.

(3) Direkt betroffen ist dagegen der Modus commercial presence, dessen Anteil die WTO auf etwa $50 \%$ schätzt, und

(4) der Modus presence of natural persons, der von der WTO nur mit $1-2 \%$ veranschlagt wird; im innereuropäischen Handel ist dieser Anteil sicher höher. Dennoch gilt, dass es bei vielen Dienstleistungen - selbst bei einem völlig diskriminierungsfreien Marktzugang - einfach nicht sinnvoll ist, größere Entfernungen und sprachliche Barrieren etc. zu überwinden.

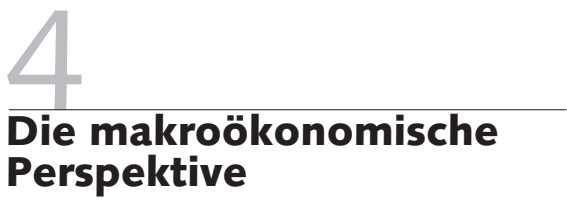

In der Diskussion um die Dienstleistungsrichtlinie wurde die Studie von Copenhagen Economics (Jensen et al. 2005) sehr häufig zitiert, besonders von Befürwortern der Richtlinie. Allerdings bezieht sich diese Studie nur auf gut zwei Drittel des von der Richtlinie betroffenen Teils der Dienstleistungswirtschaft, nämlich auf regulated professions (Steuer-, Rechts- und Wirtschaftsberatung, Buchführung), unternehmensbezogene Dienstleistungen (IT, Personaldienstleistungen, Gebäudereinigung,
Sicherheit etc.) und Handel; es fehlen Dienstleistungen aus dem Bereich der Bauwirtschaft sowie die meisten personenbezogenen Dienstleistungen, wie beispielsweise aus dem Bildungsbereich.

Die Analyse von Copenhagen Economics erfolgt in drei Schritten. Im ersten Schritt wird - wie in der oben angeführten CPB-Studie von Kox et al. (2004) - versucht, Informationen über de facto-Barrieren des Marktzutritts und internationalen Handels in Indizes umzusetzen. Jensen et al. (2005) stützen sich hier auf öffentlich zugängliche Daten, unter anderem auf die OECD-Daten, die auch vom CPB verwendet wurden. Während diese Informationen überwiegend objektiven Charakter haben, fließen bei der Konstruktion der Indizes notwendigerweise auch subjektive Einschätzungen ein, denn die Barrieren für den Austausch von Dienstleistungen müssen ausgewählt und gewichtet werden. Ein weiteres Problem sind fehlende Informationen; in diesem Fall wird davon ausgegangen, dass die entsprechende Barriere nicht existiert.

Jensen et al. (2005) errechnen jeweils einen Index für Marktzutrittsbarrieren, die für alle Anbieter zutreffen, und einen für ausländische Unternehmen. Der Unterschied zwischen diesen beiden Indizes ist ein Maß für die aktuelle Diskriminierung,

\footnotetext{
2 Als Handelshemmnis wird von Kox/Lejour/Montizaan (2004) die Unterschiedlichkeit von Regulierungen in den beiden jeweils betrachteten Ländern gewertet. In bestimmten Fällen mag das plausibel sein: Ist das Marketing an die Werberegulierung des Inlandes angepasst, so kann das Marketingkonzept ohne Anpassungskosten auf Exportmärkte übertragen werden, soweit dort ähnliche Regulierungen bestehen. Es trifft jedoch für die meisten anderen Regulierungen nicht zu, dass es in erster Linie auf die Heterogenität ankommt. Ein Beispiel sind die One-Stop-Shops, die einheitlichen Ansprechpartner für ausländische Dienstleistungsanbieter in allen bürokratischen Angelegenheiten. $\mathrm{Da}$ es diese derzeit weder in Deutschland noch in Belgien gibt, besteht keine Heterogenität. Nach Umsetzung der Dienstleistungsrichtlinie wird es One-Stop-Shops in beiden Ländern geben, was letztlich keine Veränderung der Heterogenität herstellt. Gleichwohl spielt es für den Handel mit Dienstleistungen eine große Rolle, welche Art von „Homogenität “ vorherrscht - mit oder ohne Handelshemmnisse.

3 de Bruijn/Kox/Lejour (2006) versuchen, den Einfluss, den die Aufgabe des Herkunftslandprinzips hat, zu quantifizieren.

4 Quelle: WTO Secretariat, 2005, zit. nach Mireille Cossy: Cross-Border Supply of Services - Pattern of specific commitments, http://www.wto.org/ english/tratop_e/serv_e/sym_april05_e/cossy_ e.ppt.
} 
die durch die Dienstleistungsrichtlinie abgebaut werden soll. Allerdings erfassen sie nur institutionelle Markteintrittsbarrieren; Entfernungen und sprachliche Unterschiede, die in der CPB-Studie eine entscheidende Rolle spielen, sind hier nicht explizit berücksichtigt. Im Vergleich zu den von Copenhagen Economics untersuchten Dienstleistungen sind die Rechts-, Steuerund Wirtschaftsberatung sowie die Buchführung besonders stark reguliert.

In einem zweiten Schritt versuchen Jensen et al. (2005, S. 55) die ,tariff equivalents“ zu errechnen. Preis-Kosten-Spannen und andere Indikatoren auf Unternehmensebene werden auf die Indizes regressiert; dies ermöglicht eine Prognose darüber, zu welchen Kostensenkungen eine Reduzierung der Handelshemmnisse durch die Dienstleistungsrichtlinie führt.

Im dritten und letzten Schritt werden die Auswirkungen der neuen - niedrigeren - Preise mit Hilfe eines „Rechenbaren Allgemeinen Gleichgewichtsmodells" (CGE) abzuschätzen versucht. Notwendigerweise erfordert dies über ökonometrische Schätzungen hinaus weitergehende Annahmen, die nur teilweise offengelegt werden (Jensen et al. 2005, insbes. S. 36 und Anhang B). Auch in diesem Fall sind bestimmte Sekundäreffekte ausdrücklich einbezogen, zwar nicht die direkten Folgen bürokratischer Vereinfachungen für alle Sektoren ob unter die Richtlinie fallend oder nicht -, wohl aber die Wirkungen der niedrigeren Preise für Dienstleistungs-Inputs (Jensen et al. 2005, S. 23) und auch die der größeren Angebotsvielfalt, von der die Dienstleistungsnachfrager profitieren (ebd. S. 28). ${ }^{5}$

EU-weit wird eine Zunahme um 600.000 Beschäftigte (etwa $0,3 \%$ ) angenommen, die erwartete prozentuale $\mathrm{Zu}$ nahme in Deutschland ist ähnlich hoch ${ }^{6}$ (absolut 100.000 neue Arbeitsplätze). Breuss/Badinger (2005) kombinieren die Ergebnisse des oben vorgestellten Gravitationsmodells von Kox/Lejour/Montizaan (2004) mit eigenen Schätzungen über die Wirkung verstärkten Handels auf den Wettbewerb: Je besser der Marktzugang für die ausländische Konkurrenz, desto geringer die Preis-Kosten-Spanne. Hieraus folgen - basierend auf weiteren Schätzungen - positive Wirkungen auf Produktivität, Investitionen und Beschäftigung, in einer ähnlichen Größenordnung wie bei Copenhagen Economics, für Deutschland im Bereich zwischen 77.000 und 153.000 neuen Arbeitsplätzen.
Dass mit dieser Spannbreite die notwendige Unsicherheit der Schätzung offensichtlich wird, ist sehr zu begrüßen. Darüber hinaus ist aber unklar und kaum abzuschätzen, in welchem Ausmaß die neuen Arbeitsplätze von Personen eingenommen werden, die ihre Arbeitskraft bisher schon auf dem deutschen Markt anboten, und in welchem Ausmaß aus ausländischen Arbeitsmärkten nach Deutschland gependelt oder migriert wird.

\section{Die verteilungspolitische Perspektive}

Im vorigen Abschnitt wurde aus einer makroökonomischen Perspektive argumentiert. Danach profitieren von der Dienstleistungsrichtlinie diejenigen, die Dienstleistungen nachfragen, ebenso wie die Gruppe der Dienstleister insgesamt. Beides wird möglich durch die bessere Ausnutzung von Spezialisierungsvorteilen. Anpassungen an effizientere Spezialisierungsmuster bringen es aber mit sich, dass es auch Verlierer geben muss: Das sind deutsche Dienstleistungsbranchen, in denen relativ viele Anbieter dem zunehmenden Wettbewerbsdruck nicht standhalten können. Kaum ein Ökonom wird bezweifeln, dass die Gewinner die Verlierer entschädigen könnten - aber diese Überlegung ist ein hypothetisches Konstrukt der Wohlfahrtsökonomik. Es ist durchaus möglich, dass die verteilungspolitischen Instrumente, die es gibt, etwa staatliche Lohnzuschüsse oder negative Einkommensteuer, unter den gegebenen institutionellen und polit-ökonomischen Bedingungen nicht effizient umzusetzen sind. Wer dies befürchtet, aus dessen Sicht kann die Dienstleistungsrichtlinie ein verteilungspolitisches Problem sein. Dies gilt insbesondere dann, wenn man sich anschaut, welche Branchen und welche Arbeitnehmer es sind, die mehr zu befürchten als zu erhoffen haben.

Einen ersten Hinweis gibt die - wenngleich grobe - sektorale Differenzierung der oben besprochenen Studie von Copenhagen Economics. Darin wird für Deutschland die deutlichste Zunahme der Beschäftigung für Rechts-, Steuer- und Wirtschaftsberatung etc. sowie für den Handel erwartet $(+1,4 \%$ bzw. $+2,1 \%)$; in den anderen Sektoren ist die Wirkung kaum spürbar. In den hoch qualifizierten Bera- tungsdienstleistungen können Lohnkostennachteile, wie sie in Deutschland gegenüber den östlichen (nicht aber gegenüber den westlichen) Nachbarn bestehen (IMK 2006), durch Produktdifferenzierungsvorteile wettgemacht werden. $\mathrm{Zu}$ den Gewinnern dürften nach diesem Kriterium eher Branchen mit wenigen niedrig qualifizierten Mitarbeitern gehören. Bei unternehmensbezogenen Dienstleistungen ist der höchste Anteil von Unternehmen mit einer Bruttolohnsumme über $50.000 €$ je Mitarbeiter zu finden, im Vergleich zu Bergbau/Energiewirtschaft, Industrie, Handel, Verkehr-/Nachrichtenübermittlung, Telekommunikation, Kredit- und Versicherungsgewerbewesen, die bis auf den Handel allerdings nicht von der Dienstleistungsrichtlinie betroffen sind. Relativ am höchsten sind bei den unternehmensbezogenen Dienstleistungen auch die Pro-KopfAusgaben für Weiterbildungsmaßnahmen (Alewell/Koller 2001), was ebenfalls auf hohe Humankapitalintensität schließen lässt. Wieso der deutsche Handel nach der Studie von Copenhagen Economics auch in besonderem Maße zu den Gewinnern zählen wird, ist nicht so klar. Ein Grund könnte der hohe Anteil an Kapitalkosten sein. Nerb et al. (2006) und Deutsch et al. (2006) sehen solche Dienstleistungsbranchen, die dagegen einen hohen Arbeitskostenanteil und einen geringen Anteil an hoch qualifizierter und differenzierter Arbeit aufweisen, durch zunehmenden internationalen Wettbewerb als potenziell besonders gefährdet. Allerdings muss man bedenken, dass ein Teil dieser Branchen durch die geringe Handelbarkeit vieler Dienstleistungen geschützt ist. Um dieses Kriterium quantifizieren zu können, haben wir für 70 von der Dienstleistungsrichtlinie betroffene Branchen ih-

\footnotetext{
5 In Geldeinheiten gerechnet wird eine Zunahme der Wohlfahrt um 37 Mrd. $€$ erwartet, alternative (methodisch noch gewagtere) Schätzungen von O'Toole (2005) belaufen sich auf 44 Mrd. $€$ bei weiterer Abgrenzung des betrachteten Dienstleistungssektors.

6 Ein Grund dafür dürfte sein, dass Deutschland im Ranking nach den institutionellen Markteintrittsbarrieren für Dienstleister nach den von Copenhagen Economics gebildeten Indizes im (unteren) Mittelfeld liegt und damit eine etwa durchschnittliche Wirkung der Dienstleistungsrichtlinie erfahren würde. Frühere Ergebnisse aus der Literatur, die bei Jensen et al. (2005, S. 75f.) referiert werden, sahen noch besonders hohe Barrieren für den Markteintritt in Deutschland und kommen noch zu anderen Werten.
} 
re regionale Verteilung innerhalb Deutschlands untersucht (ähnlich, aber für nur 17 Dienstleistungsbranchen: Keilbach 2002). „Die Ausgangshypothese dabei ist, dass immer dann, wenn ein Dienstleistungssektor stärker regional konzentriert ist als der potenzielle Absatzmarkt, ein überregionaler Austausch der Leistung stattfinden muss“ (Gornig/von Einem 2000, S. 66).

Zumindest bei der von uns verwendeten sektoralen Abgrenzung konnte in Deutschland keine Branche identifiziert werden, die zur höchstmöglichen Risikokategorie gehört - das wäre dann der Fall, wenn sie sowohl humankapitalarm und arbeitsintensiv ist als auch augenscheinlich über große Entfernungen handelbar. Es gibt aber Branchen, in denen Arbeitskostenunterschiede eine relativ große Rolle spielen, und die soweit gehandelt werden, dass neue ausländische Konkurrenz zumindest in Grenzregionen bedeutsam werden kann. Dazu gehören beispielsweise Gebäudereinigung, Kfz-Reparatur und Teile des Baugewerbes (für Details vgl. Nerb et al. 2006, S. 110f.). Besonders positive Wirkungen der Dienstleistungsrichtlinie aufgrund der Humankapitalcharakteristika und der Handelbarkeit - ergeben sich dagegen für deutsche Anbieter von EDVDienstleistungen, physikalischen/chemischen Analysen, Werbung, Unternehmensberatung, Forschung und Entwicklung sowie anderen wissensbasierten Dienstleistungen.

Besondere Chancen sehen Cezannel Weber (2005, S. 130) bei der „Entwicklung komplexer vernetzter Systeme von Warenproduktion und Dienstleistung, die sich der raschen Standardisierbarkeit und Verlagerbarkeit entziehen.“ Das wäre jedoch nichts Neues: Produktbegleitende Dienstleistungen wie etwa Wartung, Reparatur oder Schulungen im Zuge des Exports einer Fertigungsanlage gewinnen an Bedeutung; die Elektrotechnische Industrie erzielt mit produktbegleitenden Dienstleistungen bereits einen Umsatzanteil von fast
$20 \%$ (Nerb et al. 2006, Kap. 2.2.3.1.). Über den Außenhandel mit diesen Dienstleistungen ist allerdings so gut wie nichts bekannt, da sie statistisch nicht separat erfasst werden.

Insgesamt ist in sektoral differenzierter Betrachtung zu erwarten, dass in Deutschland besonders solche Branchen unter Druck geraten, in denen Dienstleistungen arbeitsintensiv, aber gleichzeitig wenig humankapitalintensiv produziert werden. Besondere zusätzliche Chancen ergeben sich dagegen dort, wo Arbeitskräfte bereits jetzt relativ knapp und gut bezahlt sind. Spezialisierungsgewinne durch internationalen Handel erlaubten zwar theoretisch die Entschädigung der Verlierer durch die Gewinner, das heißt aber nicht, dass in der Praxis effiziente Instrumente zum Ausgleich zwischen diesen Gruppen zur Verfügung stehen und eingesetzt werden. Insofern ist es verständlich, wenn ver.di (2006, S. 13) den Hinweis darauf, dass es in Spezialisierungsprozessen notwendigerweise neben Gewinnern auch Verlierer gibt, unbefriedigend findet. In den bisher vorliegenden Studien vermisst ver.di offensichtlich die sektoral differenzierte Analyse der verschiedenen Lohn- und Beschäftigungswirkungen. So eine Analyse dürfte allerdings schwerlich verlässlich zu leisten sein; warum das so ist, beleuchtet der abschließende Abschnitt.

\section{Fazit}

Es besteht kein ernsthafter Zweifel daran, dass mit diskriminierungsfreiem internationalen Handel von Dienstleistungen für die Volkswirtschaft - für die Gruppen aller Konsumenten und Produzenten - viel gewonnen wäre. Allerdings handelt es sich bei dieser Aussage um komparative Statik. Es werden sich neue Spezialisierungsmuster in der internationalen Arbeitsteilung bilden, aber wie diese genau aussehen und wer
Anpassungskosten in welcher Höhe tragen muss, darüber können wir wenig wissen. Das liegt daran, dass direkte Prognosemethoden nicht anwendbar sind. Im Idealfall misst man eine abhängige (,zu erklärende") Variable und eine oder mehrere erklärende Variablen und stellt fest, welcher Zusammenhang zwischen diesen besteht. Ändert sich nun eine erklärende Variable, so wird vermutet, dass der geschätzte Zusammenhang auch weiterhin besteht. Das wiederum erlaubt, Schlussfolgerungen bezüglich des Wertes der abhängigen Variablen zu ziehen.

Im Fall der Dienstleistungsrichtlinie wird eine erklärende Variable, nämlich die Höhe der Handelshemmnisse, besonders niedrige Werte annehmen - niedriger als sie je waren. Das macht direkte Prognosen auf Basis einer einfachen Regressionsgleichung fast unmöglich. So könnte es beispielsweise sein, dass nach Inkrafttreten der Dienstleistungsrichtlinie Formen des internationalen Dienstleistungshandels gefunden werden, die heute noch nicht beobachtet werden. Das Problem lässt sich mit einer einfachen Analogie veranschaulichen: Wenn wir die Menge Wasser, mit der wir eine Pflanze gießen, wöchentlich zwischen ein bis zwei Litern variieren, dann können wir die Auswirkungen auf das Wachstum noch so genau beobachten, wir wissen nicht, was passiert, wenn wir erstmals mit fünf Litern gießen. Dieses Problem kennen natürlich auch die Autoren, die versucht haben, die Folgen der Dienstleistungsrichtlinie abzuschätzen. Ihre Ansätze sind daher weniger direkt, man könnte auch sagen „tricky“. Die verbleibenden Probleme beziehen sich auf die kurz- und mittelfristigen Anpassungen. Für die lange Frist bestehen weniger Zweifel: In zehn Jahren wird sich niemand mehr die gegenwärtigen Hemmnisse für den Dienstleistungshandel zurückwünschen; die längst gefallenen Barrieren im innereuropäischen Güterhandel will schließlich auch niemand wieder aufrichten. 
Alewell, D./Koller, P. (2001): Finanzierung und Sicherung von Weiterbildungsinvestitionen, in: Personalführung 10, S. 62-71

Breuss, F./Badinger, H. (2005): The European Single Market for Services in the Context of the Lisbon Agenda: Macroeconomic Effects. Study commissioned by the Federal Ministry of Economics and Labour of the Republic of Austria, Final Report, December 2005, abridged report in: Austrian Federal Ministry of Economics and Labour (ed.): Deepening the Lisbon Agenda: Studies on Productivity, Services and Technologies http://ec.europa.eu/internal_market/services/docs/servicesdir/studies/ deepening_lisbon_studies_bmwa_vienna_2006_en.pdf

Cezanne, W./Weber, L. (2005): Die Europäische Dienstleistungsrichtlinie - Chancen und Risiken für die deutsche Wirtschaft, BTU Cottbus: Forum der Forschung 18, S. 127-132 http://www.tucottbus.de/cms/ fileadmin/ uploads_pressestelle/Forum_der_Forschung/Heft_18/Cezanne_ Weber.pdf

de Bruijn, R./Kox, H./Lejour, A. (2006): The trade-induced effects of the Services Directive and the country of origin principle, CPB Document 108 Deutsch, K. G./Gornig, M./Frank, B. (2006), The EU Services Directive: Nightmare or opportunity? Implications for transatlantic business. AICGS Policy Report 25, John Hopkins University, Washington http://www. aicgs.org/documents/polrep25.pdf

Eichenhofer, E. (2006): Stellungnahme zur Öffentlichen Anhörung: „EUDienstleistungsrichtlinie" am 16. Oktober http://www.bundestag.de/ ausschuesse/a09/anhoerungen/2_Anhoerung/Stellungnahmen/ Prof__Dr_Eichenhofer.pdf

Europäische Kommission (2002): Bericht der Kommission vom 30.07.,

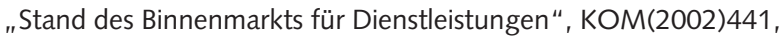
http://www.aus-portal.de/aktuell/gesetze/media/KomBerichtKOM_ 2002_441.pdf

Gornig, M./von Einem, E. (2000): Charakteristika einer dienstleistungsorientierten Exportbasis, in: Bullinger, H.-J./Stille, F. (Hrsg.): Dienstleistungsheadquarter Deutschland, Wiesbaden, S. 49-73
Institut für Makroökonomie und Konjunkturforschung (IMK) (2006): Arbeitskosten in Deutschland bisher überschätzt. Auswertung der neuen Eurostat-Statistik, IMK Report 11

Jensen, J./Svensson, P./Hvidt Thelle, M./Garcia, J./Termansen, L./ Dalgaard, T./Ballebye Olesen, H./Kastberg-Nielsen, C. (2005): Economic Assessment of the Barriers to the Internal Market for Services, Copenhagen Economics

Keilbach, M. (2002): Determinanten der räumlichen Konzentration von Industrie und Dienstleistungsbranchen. Eine empirische Analyse für Westdeutschland, in: Jahrbuch für Regionalwissenschaft 22, S. 61-79

Kox, H./Lejour, A./Montizaan, R. (2004): The free movement of services within the EU, CPB Document 69

Lorenz, F./Wannöffel, M. (2006): Besser, aber nicht gut, in: Mitbestimmung 11, S. 15-19

Nerb, G./Schmalholz, H./Frank, B./Gornig, M./Krämer, H. (2006): Chancen und Risiken veränderter Rahmenbedingungen für die Dienstleistungsunternehmen durch die EU-Dienstleistungsrichtlinie, ifo Forschungsberichte 29, München

O'Toole, R. (2005): The Services Directive - An Initial Estimate of the Economic Impact on Ireland, Forfás http://www.entemp.ie/trade/ marketaccess/singlemarket/05serv099.doc

ver.di (2006): Stellungnahme der Vereinten Dienstleistungsgewerkschaft ver.di zur Anhörung des Deutschen Bundestages, Ausschuss für Wirtschaft und Technologie zur EU-Dienstleistungsrichtlinie am 16. Oktober 2006 http://www.bundestag.de/ausschuesse/a09/anhoerungen/ 2_Anhoerung/Stellungnahmen/verdi.pdf World Trade Organization (WTO) (2001): GATS: Facts and Fiction, http://www.wto.org/english/tratop_e/serv_e/gatsfacts1004_e.pdf 